
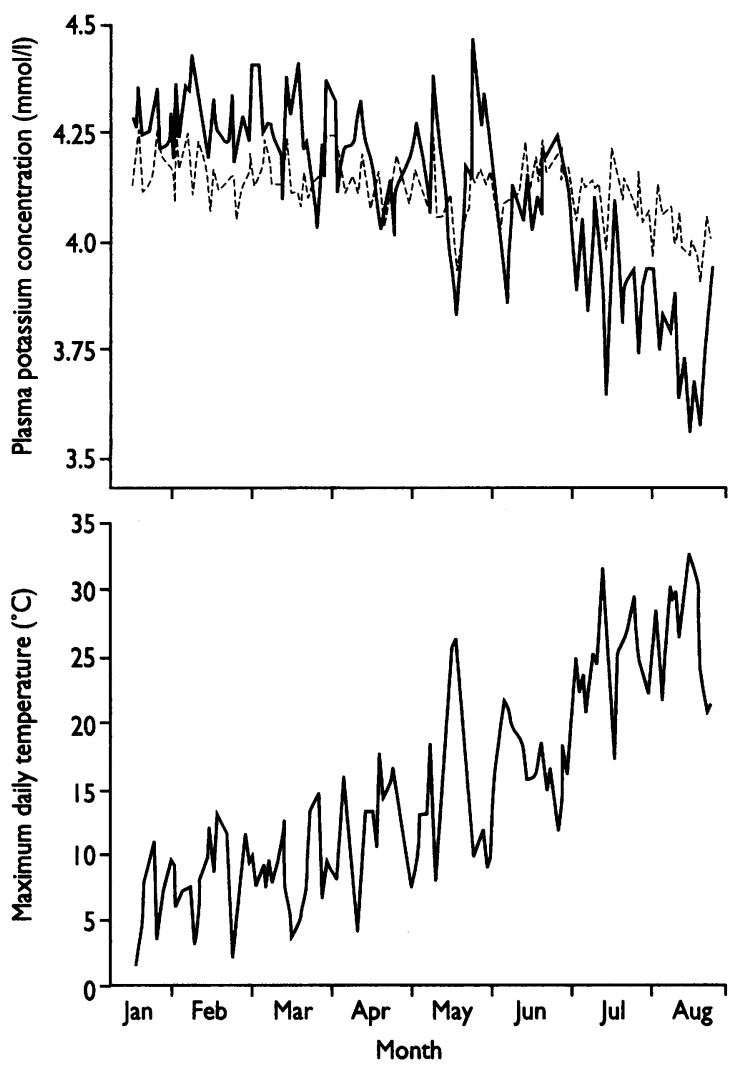

Fig 1-(Top) daily mean potassium concentrations from hospital patients (dotted line) and patients from general practice (solid line); (bottom) maximum daily temperature considered in the differential diagnosis of hypokalaemia. Laboratories and clinicians should be aware of this phenomenon so that results may be confirmed before starting unnecessary investigations and treatment. The corollary is that there may also be an increased prevalence of pseudonormokalaemia in patients who have true hyperkalaemia.

The apparent stability of potassium at $23^{\circ} \mathrm{C}$ is in agreement with a previous study which reported no change over 16 hours at $18^{\circ} \mathrm{C} .^{3}$ Another report indicates, however, that potassium concentration may fall by a mean of $0.22 \mathrm{mmol} / \mathrm{l}$ in 2 hours at $25^{\circ} \mathrm{C} .{ }^{4}$ In vitro changes in potassium are more complex than is usually stated in textbooks. ${ }^{5}$ The exact timing and temperature are clearly critical in determining the result that is actually reported by the laboratory.

We thank the laboratory staff of the department of clinical chemistry for volunteering as subjects and for performing the potassium measurements.

Funding: None.

Conflict of interest: None.

1 Goodman JR, Vincent J, Rosen I. Serum potassium changes in blood clots. Am $\mathcal{F}$ Clin Pathol 1954;24:111-3.

Moore $D$, Walker $P$, Ismail $A$. The alteration of serum potassium level during sample transit. Practitioner 1989;233:395-7.

3 Verrensen L, Lins RL, Neels H, De Broe ME. Effects of needle size and storage temperature on measurements of serum potassium. Clin Chem 1986;32:698-9.

$4 \mathrm{Kalsheker} \mathrm{N}$, Jones $\mathrm{N}$. Inaccurate in vivo plasma potassium measurements due to in vitro changes in unseparated blood. Clin Chem 1984;30:1581-2.

5 Tietz NW, Pruden EL, Siggaard-Andersen O. Electrolytes, blood gases and acid-base balance. In: Tierz NW, ed. Textbook of clinical chemistry Philadelphia: Saunders, 1986:1176-7.

(Accepted 4 March 1996)

\section{Commentary: Replication of results}

\author{
M D Buckley-Sharp, D A Gardner
}

The phenomenon of high ambient temperature causing a factitious hypokalaemia is not described in the usual textbooks and seems to be largely unknown. If it is so easily demonstrated, even in a temperate climate, then it should be equally easily repeatable.

We recovered all plasma potassium results from 1 January to 30 September 1995, divided them into two groups according to their origin (general practice and other), and calculated the mean and standard deviation for each group for each day. There were no results from general practice for Saturdays, Sundays, or holidays, and samples with these dates were removed for both groups. Over the 189 working days, there remained 6191 results from general practice and 81496 results from other sources. After reviewing the data, we thought that the clearest presentation would be given by graphing the five day rolling average of the daily means, and these are shown in figure 1.

The important features of these results are all in agreement with Masters et al. Results from general practice were usually higher than results from other sources. From late June there was a steady fall in the mean of results from general practice. By late July, mean results from general practice were lower than mean results from other sources. As our data series is longer than that of Masters et al, we observed a return towards the more usual comparison by late September. The difference in spring, and the amount of the summer reduction in results from general practice, are both quantitatively similar to the results of Masters et al. Our results were obtained with three separate ISP1000 analysers, and the changes cannot be explained by standardisation or quality variation.

It is supposed that the lowered plasma potassium concentration is caused by high temperature incubation of the samples, especially in a closed van on a hot day. Some of our samples in the general practice group did not arrive by van, while some samples from other sources did. This may explain some correlation between the two data series-as seen in August, for example.

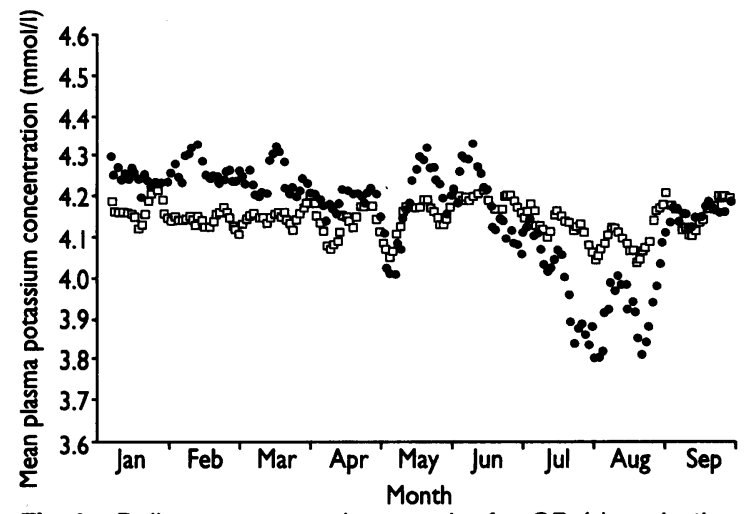

Fig 1-Daily mean potassium results for GP $(\bullet)$ and other sources (D) samples, omitting Saturdays, Sundays, and holidays, shown as five day rolling averages, 1 January to 30 September 1995 